\title{
On Becoming Editor-in-Chief of JACM
}

Becoming editor-in-chief of $J A C M$ is quite an honor. It is also a major responsibility. Let me briefly explain why I accepted this responsibility and, in the process, outline my goals for the journal.

Twenty years ago, it was possible to have a fairly good understanding of what was happening in most areas of computer science. With the explosive growth we have seen in the field in the past few decades, this is certainly not possible any more. To make matters worse, the field seems to have become more parochial. Each year we see more and more specialized conferences and workshops. It is becoming more difficult to talk technically to people who are not working in the same sub-sub-specialty that you are in. Of course, this is a problem that is not unique to computer science; it seems to be common in all scientific disciplines. This is particularly discouraging at a time when it seems that many of the most exciting advances will come from interdisciplinary work.

Some efforts at overcoming this parochialism are evident. Indeed, much of the "action" today is happening at the boundaries, both within computer science itself-work at the interfaces of programming languages, operating systems, and security, to mention just one example of many-and the boundary between computer science and other disciplines-computational biology is a prime example, with its need for algorithms, massive parallelism, scientific computing, and databases. I think that one of the best ways to help combat the fragmentation and encourage interaction is to have a journal that can provide coverage of the most significant work going on in computer science, broadly construed-our analogue of Science or Nature. That is what I would like $J A C M$ to be. This means bringing together the best articles in computer science in a timely fashion, explained in language at least accessible to other researchers.

Does this goal make sense in the brave new world of the web? I think so. I don't pretend to know the full, eventual impact of the web on scholarly publishing; I don't think anyone does. But there is no doubt that the impact will be significant. A growing number of journals are available on the web, including $J A C M$ (as of May 1997; see http://www.acm.org/dl). I expect that eventually all journals will publish only electronic versions, and we will have digital libraries that give us access to all of them. (ACM, for example, is building a digital library of all ACM publications, which members will be able to search.) Once this happens, the boundaries between journals will become fuzzier. Preprint services, which are just over the horizon, may well make the line between journal articles and preprints fuzzier. No matter how fuzzy the boundaries become, I think there will be a significant role for $J A C M$. Indeed, with the glut of information available, it will be more important than ever to have an authoritative, identifiable source of high-quality, refereed papers that provide a snapshot of the best research going on in computer science.

I said above that I would like $J A C M$ to cover "computer science, broadly construed." $J A C M$ is already trying to cover large parts of computer science; just look at the list of areas covered by our editors. However, while $J A C M$ is viewed by many as the journal of record for the theoretical computer science community, it currently does not have the same central role in all communities within 
computer science. I would like to change that. As a first step, I have added three new areas to our coverage:

—Machine Learning and Computational Learning Theory_edited by Michael Kearns

—Database Systems, Networks, and Digital Libraries—edited by Hector GarciaMolina

—Decisions, Uncertainty, and Computation-edited by Eric Horvitz

$J A C M$ also recently added another area: Computing in Technology and the Sciences. This area explicitly focuses on interdisciplinary research and applications to scientific disciplines such as biology, chemistry, and medicine, and engineering disciplines such as construction and manufacturing, technical planning, robotics, telecommunication, and circuit design. (See http://www.acm.org/ $\mathrm{jacm} /$ areas/cts.html for more details.)

Of course, just adding the coverage is not enough by itself. We hope and expect to attract the very best papers in these areas as well. It is of the utmost importance to me that $J A C M$ continue with its policy of accepting only the best papers in each of the areas that it covers.

Why should authors in, say, systems, numerical analysis, or AI publish in $J A C M$ rather than the leading specialty journals in their area? The main reason is that they can reach a far broader audience: $J A C M$ has the largest circulation of any ACM technical journal and, because of its reputation, is perused more than most computer science journals. By publishing in $J A C M$ authors will be in the best position to have their work influence a wide range of other activities. Appeal to a broad audience has always been a criterion for publication in $J A C M$. I have asked the editors to enforce this criterion, and ensure that papers accepted to JACM be written in such a way that a nonspecialist can read and understand the main ideas.

There is a secondary, but still, I think, important reason to consider JACM: price. Journal pricing is not an issue that authors or journal editors have traditionally been concerned with, but times are changing. Anyone who has spoken to a librarian recently knows that the prices of most journals have increased at a rate several times that of inflation, while most library budgets have barely kept up with inflation. As a result, libraries are being forced to delete journals. $J A C M$ is less than one-quarter of the price of typical journals published by for-profit publishers. I believe that the community should make a special effort to try to support reasonably-priced journals.

A prerennial area of concern for readers, authors and editors is time to publication. This has been a problem for $J A C M$ in the past; I am optimistic that the worst is now over. I will work with the editorial board to speed up the review process as much as possible. I plan to decentralize the submission procedure (so that authors can submit directly to the cognizant editor), to avoid bottlenecks in my office. In addition, I believe we can achieve significant speed-ups just by using tools already available. Electronic submission, which is now encouraged, will help by cutting down communication time between authors, editors, and referees. papers will be posted on the $J A C M$ website as soon as they are accepted (provided that authors submit an electronic version). (See http://www.acm.org/ jacm/authors.html for details on electronic submission and publication.) Given 
our current low backlog (1-2 issues), and the fact that $J A C M$ is now published six times a year, the time between acceptance and print appearance should also be short.

Let me conclude by welcoming some new faces to the editorial board. I have already mentioned Eric Horvitz, Hector Garcia-Molina, and Michael Kearns. Due to the number of submissions in the area of Parallel Computation, I have subdivided it into Parallel Algorithms, to be edited by Vijaya Ramachandran, and Parallel Computing, to be edited by Guy Blelloch; the former focuses more on theoretical, algorithmic issues, while the latter focuses more on architectural and systems issues. (Obviously, there is not a sharp division between the areas.) Hal Gabow will be taking over as area editor for Data Structures and Analysis of Algorithms and Johan Hastad for Formal Languages and Complexity Theory. There are also three other relatively recent additions to the board that should be mentioned: Bernard Chazell, Robert Harper, and Robert Schreiber, who edit Computational Geometry, Programming Languages and Methodology, and Scientific Computing, respectively. I would like to thank all the new and continuing members of the board for agreeing to serve. I would also like to thank the departing members of the editorial board-Michael Sipser, Robert Sedgwick, and Lawrence Snyder, and especially Tom Leighton, the previous editor-inchief-for their years of dedicated service.

$J A C M$ will continue to prosper only with your support. Please send me your suggestions and comments any time. 